\title{
Comparative Study between Dexmedetomidine and Dexamethasone as an Adjuvant to Bupivacaine in Ultrasound Guided Supraclavicular Brachial Plexus Block in Upper Limb Surgeries
}

\author{
Mohamed Hussien Hamada, Wael Mohamed Elmahdy Ibrahim, Mohamed Adel Ashiry \\ Department of Anesthesia and ICU, Faculty of Medicine, Al-Azhar University, Cairo, Egypt \\ Corresponding author: Mohamed Adel Ashiry, Mobile: 01004814878, email: ashirybeng@ gmail.com
}

\begin{abstract}
Background: Brachial plexus block is frequently used for ambulatory upper limb surgery. It can significantly reduce pain and nausea, allowing for faster discharge from hospital when compared with general anesthesia (GA). Doing this block through ultrasound has higher index of safety, allowing providers to identify the appropriate point of injection and performing this block with greater accuracy under direct visualization.

Objective: the aim of the study was to compare the efficacy of bupivacaine plus dexamethasone versus bupivacaine plus dexmedetomidine as adjuvants on block characteristics including onset and duration of sensory and motor blockade, duration of postoperative analgesia, post-operative pain score and sedation score.

Patients and Methods: This prospective double - blinded, randomized controlled trial included a total of 60 patients divided to two groups, each group contained 30 patients. Group 1 (dexmedetomidine group) had received $1 \mu \mathrm{g} / \mathrm{kg}$ of dexmedetomidine $+20 \mathrm{ml}$ bupivacaine $5 \%$. Group 2 (dexamethasone group) had received dexamethasone $+20 \mathrm{ml}$ of bupivacaine $5 \%$.
\end{abstract}

Results: In this study the onset and duration of sensory and motor blockade was statistically shorter in dexamethasone group when compared to dexmedetomidine group.

Conclusion: It could be concluded that addition of dexmedetomidine to bupivacaine prolongs the time of block and analgesia duration longer than dexamethasone, but the onset of block was shorter when dexamethasone was added to bupivacaine.

Keywords: dexmedetomidine, supraclavicular block, bupivacaine, upper limb surgery.

\section{INTRODUCTION}

Regional nerve block offer alternative choice of anesthesia using minimal anesthetic drugs and high safety profile. Brachial plexus block is a popular and widely employed regional nerve block technique for perioperative anesthesia and analgesia for surgery of the upper extremity and supraclavicular approach is the easiest and most consistent method for surgery below the shoulder joint ${ }^{(1)}$.

Supraclavicular nerve block is good alternative to general anesthesia for upper limb surgery. This avoids the complication of general anesthetic drugs and upper airway intubation and its complication. It achieves complete muscle relaxation, intraoperative hemodynamic stability, and postoperative analgesia ${ }^{(2)}$.

Bupivacaine used frequently for supraclavicular nerve block as it has long duration of action from 6 to $9 \mathrm{~h}$. Adjuvant to local anesthetics for brachial plexus block may enhance the quality and duration of analgesia ${ }^{(3)}$.

Brachial plexus blockade reduces pain, but still with short duration and the challenge remains to increase the duration of analgesia with decreasing sideeffects ${ }^{(4)}$.

Different adjuvants have been used to prolong regional Blockade, shorten the onset times of blocks and prolonge duration of post operative analgesia. Various adjuvants, including opioids, midazolam, magnesium sulfate, dexamethasone, and neostigmine, have been added to local anesthetics in an attempt to increase the duration of block and postoperative analgesia with the risk of various adverse effects. The efficacy of $\alpha 2$-adrenoceptor agonists has been established in a variety of regional anesthesia techniques. Clonidine, when added to lidocaine, prolonged the duration of anesthesia and analgesia after brachial plexus block ${ }^{(4)}$.

Dexmedetomidine is a selective $\alpha 2$ adrenoceptor agonist and is approximately 8 times more potent than clonidine ${ }^{(5)}$. Dexmedetomidine is also reportedly safe and effective when administered with long-acting local anesthetics in peripheral nerve blocks $^{(6)}$. No significant histopathologic abnormalities were reported after intrathecal or perineural administration of dexmedetomidine ${ }^{(7)}$.

Various clinical trials have found that administration of dexmedetomidine with local anesthetics in neuraxial and peripheral nerve blocks prolonged the duration of sensory and motor blockade $^{(8)}$.

Steroids have powerful anti-inflammatory as well as analgesic property. They suppress inflammation through inhibition of phospholipase A2. Local application of methylprednisolone has been found to block transmission in nociceptive $\mathrm{C}$-fibers but not in myelinated A-beta fibers ${ }^{(9)}$. The effect was reversible, suggesting a direct membrane action of steroids. Corticosteroids also suppress ectopic neuronal discharge. Perineural injection of glucocorticoid along with local anesthetics is reported to influence the onset and duration of sensory and motor block ${ }^{(10)}$. Dexamethasone is a very potent and highly selective glucocorticoid. Various studies have been done using dexamethasone $4 \mathrm{mg}$ as an adjuvant to local 
anaesthetics mixture in brachial plexus block resulting in variable effects on onset but prolonged duration of analgesia and motor block ${ }^{(11)}$.

The aim of the study was to compare the efficacy of bupivacaine plus dexamethasone versus bupivacaine plus dexmedetomidine as adjuvants on block characteristics including onset and duration of sensory and motor blockade, duration of postoperative analgesia, post-operative pain score and sedation score.

\section{PATIENTS AND METHODS}

This prospective double blinded, randomized controlled trial included a total of 60 patients scheduled for upper limb surgeries not exceeding 2 hours attending at Al-Azhar University Hospitals. Approval of the ethical committee and a written informed consent from all the subjects after explaining to them the nature of study and complications were obtained. This study was conducted between March 2018 to September 2018.

Patients were divided to two groups, each group contained 30 patients: Group 1 (dexmedetomidine group) had received $1 \mu \mathrm{g} / \mathrm{kg}$ of dexmedetomidine $+20 \mathrm{ml}$ bupivacaine $5 \%$. Group 2 (dexamethasone group): received $4 \mathrm{mg}$ dexamethasone $+20 \mathrm{ml}$ of bupivacaine $5 \%$.

Inclusion criteria: Patients with American Society of Anesthesiologists (ASA) grades I and II, aged 20 to 60 years, of either sex, BMI less than 36 $\mathrm{Kg} / \mathrm{M}^{2}$ and who scheduled for elective upper limb orthopedic surgeries not exceeding 2 hours as fractures and plastic surgeries.

Exclusion criteria: Local infection at the site of puncture. Patients having any neurologic deficit in the upper limb. Patients having history of hematological disorders, including coagulation abnormality. Patient has a known allergy to study drug or additions. Pregnant women. Systemic use of corticosteroid for two weeks or longer, drug abuse.

Blinding: The local anesthetic with dexmedetomidine or with dexamethasone was prepared in syringe by another study anesthesiologist than who performed the block and assessed the pain and the effect of block.

The commercially available forms of the study drugs that were used: A $20 \mathrm{ml}$ vial of Bupivacaine $0.5 \%$ (Bupivacaine HCL) equivalent to $5 \mathrm{mg} / \mathrm{ml}$ (sunny pharmaceutical industries. Egypt). A 2 $\mathrm{ml}$ vial of Precedex (Dexmedetomedine HCL) equivalent to $100 \mathrm{mcg} / \mathrm{ml}$ (Hospira Inc, USA). A $2 \mathrm{ml}$ ampoule of dexamethasone $8 \mathrm{mg}$ (Dexamethasone sodium phosphate) equivalent to $4 \mathrm{mg} / \mathrm{ml}$ (Amriya Pharm. Ind. Egypt). Group (1): received $1 \mu \mathrm{g} / \mathrm{kg}$ of dexmedetomidine $+20 \mathrm{ml}$ bupivacaine $5 \%$. Group (2): received $4 \mathrm{mg}$ dexamethasone $+20 \mathrm{ml}$ of bupivacaine $5 \%$.
A successful block was defined as complete sensory and motor blockade in all regions of median, ulnar, and redial nerve distribution and the block were assessed every 5 minutes after local anesthetic injection.

Anesthetic failure in the surgical area was defined as if the patient experienced pain during surgery and this was controlled by IV fentanyl 1-2 $\mu \mathrm{g} / \mathrm{kg}$, or recourse to general anesthesia, those patients not included in our study and was replaced by other patients.

Surgical effectiveness was defined as surgery ended without patient discomfort and the need for supplementation of the block.

Assessment of Sensory Block: Was assessed by ice bag test by applying ice, a cold gel bag, or a wet alcohol sponge. The test was done every 5 minutes for 30 minutes and time of loss of sensation all over the forearm and hand was recorded then every 4 hours till resolution of sensory block or first analgesia rescue and the time was recorded. Duration of sensory block was defined as the time interval between the success of the block and the complete resolution of anesthesia on all nerves area and it was recorded.

Assessment of Motor block: Motor block was determined by thumb abduction (radial nerve), thumb adduction (ulnar nerve), thumb opposition (median nerve), and flexion of elbow (musculocutaneous nerve) according to the modified Bromage scale:

Duration of motor block was defined as the time interval between the success of the block and the recovery of complete motor function of forearm and hand.

Assessment of sedation: Sedation was assessed to the patients after administration of drugs every 30min in first two hours then every 2 hours till 6 hours post operative using Ramsey sedation scale.

Hemodynamic parameters: On arrival to the operating theater, the vital signs parameters including MAP, HR, RR and $\mathrm{SpO}_{2}$ was recorded at base line, 15, 30, 45, 60, $120 \mathrm{~min}$.

Post-operative settings: At the end of the surgery, the patient was kept under observation postoperatively for 1 hour to monitor vital signs (conscious level, blood pressure, heart rate, respiratory rate and pattern) then discharged to ward to be followed for returning of pain.

Side effect and Complication assessment: The patients were observed for the occurrence of any adverse effect and/or complication related to the procedure (e.g. pneumothorax, hematoma), or to the study drugs [e.g. hypotension/hypertension (i.e. 20\% decrease or increase from the baseline value), bradycardia (HR <50 beats/min) or tachycardia (HR > 120 beats/min), nausea, vomiting, and hypoxemia $(\mathrm{SpO} 2<90 \%)]$. 
Postoperative pain: The assessment of postoperative pain was done every 2 hours in the recovery room and in surgical ward with the help of Numeric Rating Scale (1-10). At score of 4, rescue analgesic (inj. diclofenac sodium $(1.5 \mathrm{mg} / \mathrm{kg})$ intramuscularly) was given. Duration of analgesia will be the time from drug injection to the time of first rescue of analgesia. total analgesic dose during first 24 hours will be recorded.

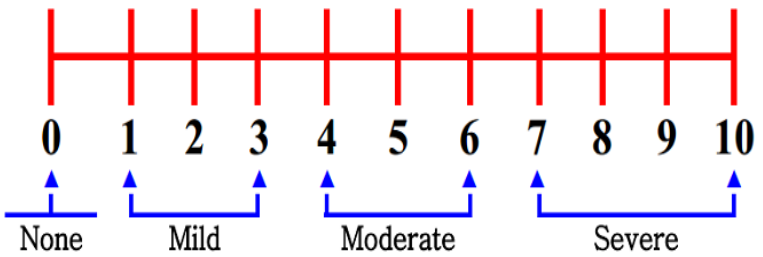

Fig. (1): Numeric rating scale. (this figure should be number 1)

Statistical Analysis

RESULTS

Table (1): Comparison between study groups as regards age, sex, ASA \& body weight variation.

\begin{tabular}{|c|c|c|c|c|c|c|}
\hline & $\begin{array}{c}\text { Dexmedetomidine } \\
\text { group }\end{array}$ & $\begin{array}{c}\text { Dexamethasone } \\
\text { group }\end{array}$ & \multirow[t]{2}{*}{ Test value } & \multirow[t]{2}{*}{$P$-value } & \multirow[t]{2}{*}{ Sig. } \\
\hline & & Mean \pm SD & Mean \pm SD & & & \\
\hline Age (years) & $\begin{array}{l}\text { Mean } \pm \text { SD } \\
\text { Range }\end{array}$ & $\begin{array}{c}36.87 \pm 11.52 \\
18-60\end{array}$ & $\begin{array}{c}35.80 \pm 10.75 \\
19-58\end{array}$ & $0.371 \bullet$ & 0.712 & NS \\
\hline Sex & $\begin{array}{l}\text { Males } \\
\text { Females }\end{array}$ & $\begin{array}{c}23(76.7 \%) \\
7(23.3 \%)\end{array}$ & $\begin{array}{l}17(56.7 \%) \\
13(43.3 \%)\end{array}$ & $2.700^{*}$ & 0.100 & NS \\
\hline ASA & \begin{tabular}{|l|}
1 \\
2 \\
\end{tabular} & $\begin{array}{c}23(76.7 \%) \\
7(23.3 \%) \\
\end{array}$ & $\begin{array}{c}21(70.0 \%) \\
9(30.0 \%) \\
\end{array}$ & $0.341^{*}$ & 0.559 & NS \\
\hline Weight & $\begin{array}{l}\text { Mean } \pm \text { SD } \\
\text { Range }\end{array}$ & $\begin{array}{c}77.97 \pm 5.85 \\
65-90\end{array}$ & $\begin{array}{c}76.50 \pm 8.89 \\
58-90\end{array}$ & $0.755^{\bullet}$ & 0.453 & NS \\
\hline
\end{tabular}

P-value >0.05: Non significant (NS); P-value <0.05: Significant (S); P-value $<0.01$ : highly significant (HS)

$*:$ Chi-square test; $\bullet:$ Independent t-test

Table (2): Comparison of duration of surgery between the study groups.

\begin{tabular}{|c|c|c|c|c|c|}
\hline \multirow{2}{*}{$\begin{array}{l}\text { Duration of surgery } \\
\text { (min) }\end{array}$} & $\begin{array}{c}\text { Dexmedetomidine } \\
\text { group }\end{array}$ & $\begin{array}{c}\text { Dexamethasone } \\
\text { group }\end{array}$ & \multirow[t]{2}{*}{ Test value• } & \multirow[t]{2}{*}{ P-value } & \multirow[t]{2}{*}{ Sig. } \\
\hline & Mean \pm SD & Mean \pm SD & & & \\
\hline $\begin{array}{l}\text { Mean } \pm \text { SD } \\
\text { Range }\end{array}$ & $\begin{array}{c}97.67 \pm 16.07 \\
70-120\end{array}$ & $\begin{array}{c}96.17 \pm 18.08 \\
60-120\end{array}$ & 0.340 & 0.735 & NS \\
\hline
\end{tabular}

P-value >0.05: Non significant (NS); P-value <0.05: Significant (S); P-value $<0.01$ : highly significant (HS)

$\because$ Independent t-test

Table (3): Comparison of onset of sensory block between the study groups.

\begin{tabular}{|l|c|c|c|c|c|}
\hline $\begin{array}{l}\text { Onset of sensory } \\
\text { block (min) }\end{array}$ & $\begin{array}{c}\text { Dexmedetomidine } \\
\text { group }\end{array}$ & $\begin{array}{c}\text { Dexamethasone } \\
\text { group }\end{array}$ & \multirow{2}{*}{ Test value } & P-value & \multirow{2}{*}{ Sig. } \\
\cline { 2 - 4 } $\begin{array}{l}\text { Mean } \pm \text { SD } \\
\text { Range }\end{array}$ & $\begin{array}{c}\text { Mean } \pm \text { SD } \\
4-43 \pm 1.33\end{array}$ & $\begin{array}{c}6.57 \pm 1.45 \\
4-10\end{array}$ & 2.408 & 0.019 & $\mathrm{~S}$ \\
\hline
\end{tabular}

P-value >0.05: Non significant (NS); P-value <0.05: Significant (S); P-value $<0.01$ : highly significant (HS)

$\because$ Independent t-test 
Table (4): Comparison of complete of sensory block between the study groups.

\begin{tabular}{|c|c|c|c|c|c|}
\hline $\begin{array}{c}\text { Complete of } \\
\text { sensory block } \\
(\mathbf{m i n})\end{array}$ & $\begin{array}{c}\text { Dexmedetomidine } \\
\text { group }\end{array}$ & $\begin{array}{c}\text { Dexamethasone } \\
\text { group }\end{array}$ & Test value & P-value & Sig. \\
\cline { 2 - 3 } & Mean \pm SD & Mean \pm SD & & & HS \\
\hline Mean \pm SD & $19.10 \pm 2.56$ & $16.57 \pm 2.65$ & 3.764 & 0.000 & Hen \\
Range & $14-24$ & $12-21$ &
\end{tabular}

P-value >0.05: Non significant (NS); P-value <0.05: Significant (S); P-value $<0.01$ : highly significant (HS)

$\because$ Independent t-test

Table (5): Comparison of duration of sensory block between the study groups.

\begin{tabular}{|l|c|c|c|c|c|}
\hline $\begin{array}{c}\text { Duration of } \\
\text { sensory block } \\
(\mathbf{h})\end{array}$ & $\begin{array}{c}\text { Dexmedetomidine } \\
\text { group }\end{array}$ & $\begin{array}{c}\text { Dexamethasone } \\
\text { group }\end{array}$ & \multirow{2}{*}{ Test value } & \multirow{2}{*}{ P-value } & \multirow{2}{*}{ Sig. } \\
\cline { 2 - 4 } & Mean \pm SD & Mean \pm SD & & & HS \\
\hline $\begin{array}{l}\text { Mean } \pm \text { SD } \\
\text { Range }\end{array}$ & $19.00 \pm 1.80$ & $\begin{array}{c}12.03 \pm 1.54 \\
9-16\end{array}$ & 16.097 & 0.000 & H \\
\hline
\end{tabular}

P-value >0.05: Non significant (NS); P-value <0.05: Significant (S); P-value $<0.01$ : highly significant (HS)

$\because$ Independent t-test

Table (6): Comparison of onset of motor block between the study groups.

\begin{tabular}{|c|c|c|c|c|c|}
\hline $\begin{array}{c}\text { Onset of } \\
\text { motor } \\
\text { block }(\text { min) }\end{array}$ & $\begin{array}{c}\text { Dexmedetomidine } \\
\text { group }\end{array}$ & $\begin{array}{c}\text { Dexamethasone } \\
\text { group }\end{array}$ & \multirow{2}{*}{ Test value } & P-value & \multirow{2}{*}{ Sig. } \\
\cline { 2 - 3 } Mean \pm SD & Mean \pm SD & Mean \pm SD & & & \\
Range & $\begin{array}{c}6.10 \pm 1.12 \\
4-8\end{array}$ & $\begin{array}{c}5.00 \pm 1.08 \\
4-8\end{array}$ & 3.859 & 0.000 & HS \\
\hline
\end{tabular}

P-value >0.05: Non significant (NS); P-value $<0.05$ : Significant (S); P-value $<0.01$ : highly significant (HS)

$\because$ Independent t-test

Table (7): Comparison of onset of motor block between the study groups.

\begin{tabular}{|l|c|c|c|c|c|}
\hline $\begin{array}{c}\text { Complete of } \\
\text { motor block } \\
(\mathbf{m i n})\end{array}$ & $\begin{array}{c}\text { Dexmedetomidine } \\
\text { group }\end{array}$ & $\begin{array}{c}\text { Dexamethasone } \\
\text { group }\end{array}$ & \multirow{2}{*}{ Test value } & P-value & \multirow{2}{*}{ Sig. } \\
\cline { 2 - 5 } & Mean \pm SD & Mean \pm SD & & & HS \\
\hline $\begin{array}{l}\text { Mean } \pm \text { SD } \\
\text { Range }\end{array}$ & $\begin{array}{c}23.47 \pm 2.67 \\
18-28\end{array}$ & $\begin{array}{c}20.57 \pm 3.22 \\
15-28\end{array}$ & 3.792 & 0.000 & HS \\
\hline
\end{tabular}

P-value >0.05: Non significant (NS); P-value <0.05: Significant (S); P-value< 0.01: highly significant (HS)

$\because$ Independent t-test

Table (8): Comparison of duration of motor block between the study groups.

\begin{tabular}{|l|c|c|c|c|c|}
\hline \multirow{2}{*}{$\begin{array}{c}\text { Duration of } \\
\text { motor block (h) }\end{array}$} & $\begin{array}{c}\text { Dexmedetomidine } \\
\text { group }\end{array}$ & $\begin{array}{c}\text { Dexamethasone } \\
\text { group }\end{array}$ & \multirow{2}{*}{ Test value } & \multirow{2}{*}{ P-value } & \multirow{2}{*}{ Sig. } \\
\cline { 2 - 6 } & Mean \pm SD & Mean \pm SD & & \multirow{2}{*}{0.000} & HS \\
\hline Mean \pm SD & $18.93 \pm 1.76$ & $\begin{array}{c}11.73 \pm 1.72 \\
9-15\end{array}$ & 16.020 & 0.000 \\
\hline
\end{tabular}

P-value >0.05: Non significant (NS); P-value <0.05: Significant (S); P-value $<0.01$ : highly significant (HS)

$\because$ Independent t-test

Table (9): Comparison of duration of analgesia between the study groups.

\begin{tabular}{|l|c|c|c|c|c|}
\hline \multirow{2}{*}{$\begin{array}{c}\text { 1st analgesia } \\
\text { rescue (h) }\end{array}$} & $\begin{array}{c}\text { Dexmedetomidine } \\
\text { group }\end{array}$ & $\begin{array}{c}\text { Dexamethasone } \\
\text { group }\end{array}$ & \multirow{2}{*}{ Test value } & \multirow{2}{*}{ P-value } & \multirow{2}{*}{ Sig. } \\
\cline { 2 - 3 } & Mean \pm SD & Mean \pm SD & & & HS \\
\hline Mean \pm SD & $19.37 \pm 1.92$ & $12.67 \pm 1.67$ & 14.389 & 0.000 & Hen \\
Range & $16-23$ & $10.2-17$ & &
\end{tabular}

P-value >0.05: Non significant (NS); P-value <0.05: Significant (S); P-value $<0.01$ : highly significant (HS)

$\because$ Independent t-test 
Table (10): Comparison of sedation score between study groups.

\begin{tabular}{|c|c|c|c|c|c|c|}
\hline & $\begin{array}{c}\text { Dexmedetomidine } \\
\text { group }\end{array}$ & $\begin{array}{c}\text { Dexamethasone } \\
\text { group }\end{array}$ & \multirow[t]{2}{*}{ Test value } & \multirow[t]{2}{*}{ P-value } & \multirow[t]{2}{*}{ Sig. } \\
\hline & & Mean \pm SD & Mean \pm SD & & & \\
\hline Sedation 0 & $\begin{array}{l}\text { Mean } \pm \text { SD } \\
\text { Range }\end{array}$ & $\begin{array}{l}2.00 \pm 0.00 \\
2-2\end{array}$ & $\begin{array}{l}2.00 \pm 0.00 \\
2-2\end{array}$ & 0.001 & 1.000 & NS \\
\hline Sedation 30mins & $\begin{array}{l}\text { Mean } \pm \text { SD } \\
\text { Range }\end{array}$ & $\begin{array}{l}2.50 \pm 0.51 \\
2-3\end{array}$ & $\begin{array}{l}2.00 \pm 0.00 \\
2-2\end{array}$ & 5.385 & 0.001 & HS \\
\hline Sedation 60mins & $\begin{array}{l}\text { Mean } \pm \text { SD } \\
\text { Range }\end{array}$ & $\begin{array}{c}2.53 \pm 0.51 \\
2-3\end{array}$ & $\begin{array}{l}2.13 \pm 0.35 \\
2-3\end{array}$ & 3.568 & 0.001 & HS \\
\hline Sedation 90mins & $\begin{array}{l}\text { Mean } \pm \text { SD } \\
\text { Range }\end{array}$ & $\begin{array}{l}2.53 \pm 0.51 \\
2-3\end{array}$ & $\begin{array}{c}2.17 \pm 0.38 \\
2-3\end{array}$ & 3.171 & 0.002 & HS \\
\hline Sedation 120mins & $\begin{array}{l}\text { Mean } \pm \text { SD } \\
\text { Range }\end{array}$ & $\begin{array}{l}2.53 \pm 0.51 \\
2-3\end{array}$ & $\begin{array}{l}2.17 \pm 0.38 \\
2-3\end{array}$ & 3.171 & 0.002 & HS \\
\hline Sedation $4 \mathrm{~h}$ & $\begin{array}{l}\text { Mean } \pm \text { SD } \\
\text { Range }\end{array}$ & $\begin{array}{c}2.53 \pm 0.51 \\
2-3\end{array}$ & $\begin{array}{c}2.13 \pm 0.35 \\
2-3\end{array}$ & 3.568 & 0.001 & HS \\
\hline Sedation $6 \mathrm{~h}$ & Mean \pm SD & $\begin{array}{l}2.53 \pm 0.51 \\
2-3\end{array}$ & $\begin{array}{l}2.07 \pm 0.25 \\
2-3\end{array}$ & 4.506 & 0.001 & HS \\
\hline
\end{tabular}

P-value >0.05: Non significant (NS); P-value <0.05: Significant (S); P-value $<0.01$ : highly significant (HS)

$\because$ Independent t-test

Table (11): Comparison of post operative pain between the study groups.

\begin{tabular}{|l|c|c|c|c|c|}
\hline $\begin{array}{c}\text { Post operative pain } \\
\text { (numeric Rating } \\
\text { Scale) }\end{array}$ & $\begin{array}{c}\text { Dexmedetomidine } \\
\text { group }\end{array}$ & $\begin{array}{c}\text { Dexamethasone } \\
\text { group }\end{array}$ & \multirow{2}{*}{ Test value } & \multirow{2}{*}{ P-value } & \multirow{2}{*}{ Sig. } \\
\cline { 2 - 3 } & Mean \pm SD & Mean \pm SD & & \multirow{2}{*}{ NS } \\
\hline $\begin{array}{l}\text { Mean } \pm \text { SD } \\
\text { Range }\end{array}$ & $\begin{array}{c}3.83 \pm 0.53 \\
3-5\end{array}$ & $\begin{array}{c}3.87 \pm 0.63 \\
3-5\end{array}$ & -0.222 & 0.825 & N \\
\hline
\end{tabular}

P-value >0.05: Non significant (NS); P-value <0.05: Significant (S); P-value $<0.01$ : highly significant (HS)

$\bullet$ Independent $t$-test

Table (12): Comparison between systolic blood pressure regarding of the two blocks in the study.

\begin{tabular}{|c|c|c|c|c|c|c|}
\hline & $\begin{array}{c}\text { Dexmedetomidine } \\
\text { group }\end{array}$ & $\begin{array}{c}\text { Dexamethasone } \\
\text { group }\end{array}$ & \multirow[t]{2}{*}{ Test value• } & \multirow[t]{2}{*}{ P-value } & \multirow[t]{2}{*}{ Sig. } \\
\hline & & Mean \pm SD & Mean \pm SD & & & \\
\hline Systolic Blood pressure 0 & $\begin{array}{l}\text { Mean } \pm \text { SD } \\
\text { Range }\end{array}$ & $\begin{array}{c}122.00 \pm 12.57 \\
105-160\end{array}$ & $\begin{array}{c}120.83 \pm 11.07 \\
105-150\end{array}$ & 0.382 & 0.704 & NS \\
\hline Systolic Blood pressure 15 & $\begin{array}{l}\text { Mean } \pm \text { SD } \\
\text { Range }\end{array}$ & $\begin{array}{c}117.17 \pm 12.71 \\
100-150\end{array}$ & $\begin{array}{c}120.00 \pm 10.75 \\
105-150\end{array}$ & -0.932 & 0.355 & NS \\
\hline Systolic Blood pressure 45 & $\begin{array}{l}\text { Mean } \pm \text { SD } \\
\text { Range }\end{array}$ & $\begin{array}{c}114.50 \pm 11.77 \\
100-140\end{array}$ & $\begin{array}{c}117.00 \pm 11.19 \\
100-150\end{array}$ & -0.843 & 0.403 & NS \\
\hline Systolic Blood pressure 60 & $\begin{array}{l}\text { Mean } \pm \text { SD } \\
\text { Range }\end{array}$ & $\begin{array}{c}112.50 \pm 10.40 \\
100-140\end{array}$ & $\begin{array}{c}114.83 \pm 11.56 \\
95-140\end{array}$ & -0.822 & 0.414 & NS \\
\hline Systolic Blood pressure 120 & $\begin{array}{l}\text { Mean } \pm \text { SD } \\
\text { Range }\end{array}$ & $\begin{array}{c}109.83 \pm 11.41 \\
90-140\end{array}$ & $\begin{array}{c}114.00 \pm 11.55 \\
90-140\end{array}$ & -1.406 & 0.165 & NS \\
\hline Systolic Blood pressure $4 \mathrm{~h}$ & $\begin{array}{l}\text { Mean } \pm \text { SD } \\
\text { Range }\end{array}$ & $\begin{array}{c}110.83 \pm 10.99 \\
90-140\end{array}$ & $\begin{array}{c}114.60 \pm 11.52 \\
90-140\end{array}$ & -1.296 & 0.200 & NS \\
\hline Systolic Blood pressure $6 \mathrm{~h}$ & $\begin{array}{l}\text { Mean } \pm \text { SD } \\
\text { Range }\end{array}$ & $\begin{array}{c}113.67 \pm 10.25 \\
90-140\end{array}$ & $\begin{array}{c}116.83 \pm 10.21 \\
105-145\end{array}$ & -1.199 & 0.235 & NS \\
\hline
\end{tabular}

P-value >0.05: Non significant (NS); P-value <0.05: Significant (S); P-value< 0.01: highly significant (HS)

$\because$ Independent t-test 
Table (13): Comparison between diastolic blood pressure regarding of the two blocks in the study.

\begin{tabular}{|c|c|c|c|c|c|c|}
\hline & $\begin{array}{c}\text { Dexmedetomidine } \\
\text { group }\end{array}$ & $\begin{array}{c}\text { Dexamethasone } \\
\text { group }\end{array}$ & \multirow{2}{*}{$\begin{array}{c}\text { Test } \\
\text { value• }\end{array}$} & \multirow{2}{*}{$\begin{array}{c}P \text { - } \\
\text { value }\end{array}$} & \multirow{2}{*}{$\begin{array}{l}\text { Sig } \\
\cdot\end{array}$} \\
\hline & & Mean \pm SD & Mean \pm SD & & & \\
\hline Diastolic Blood pressure 0 & $\begin{array}{l}\text { Mean } \pm \text { SD } \\
\text { Range }\end{array}$ & $\begin{array}{l}71.00 \pm 9.77 \\
50-100\end{array}$ & $\begin{array}{l}69.67 \pm 11.21 \\
50-95\end{array}$ & 0.491 & 0.625 & NS \\
\hline $\begin{array}{l}\text { Diastolic Blood pressure } \\
15\end{array}$ & $\begin{array}{l}\text { Mean } \pm \text { SD } \\
\text { Range }\end{array}$ & $\begin{array}{c}68.00 \pm 10.31 \\
50-100 \\
\end{array}$ & $\begin{array}{c}68.67 \pm 10.42 \\
55-95\end{array}$ & -0.249 & 0.804 & NS \\
\hline $\begin{array}{l}\text { Diastolic Blood pressure } \\
45\end{array}$ & $\begin{array}{l}\text { Mean } \pm \text { SD } \\
\text { Range }\end{array}$ & $\begin{array}{c}64.67 \pm 10.98 \\
45-90\end{array}$ & $\begin{array}{c}65.33 \pm 12.66 \\
50-90\end{array}$ & -0.218 & 0.828 & NS \\
\hline $\begin{array}{l}\text { Diastolic Blood pressure } \\
60\end{array}$ & $\begin{array}{l}\text { Mean } \pm \text { SD } \\
\text { Range }\end{array}$ & $\begin{array}{c}62.00 \pm 10.88 \\
50-95\end{array}$ & $\begin{array}{c}65.00 \pm 11.45 \\
50-90\end{array}$ & -1.041 & 0.302 & NS \\
\hline $\begin{array}{l}\text { Diastolic Blood pressure } \\
120\end{array}$ & $\begin{array}{l}\text { Mean } \pm \text { SD } \\
\text { Range }\end{array}$ & $\begin{array}{c}59.50 \pm 10.53 \\
45-90\end{array}$ & $\begin{array}{c}65.00 \pm 11.82 \\
50-90\end{array}$ & -1.903 & 0.062 & NS \\
\hline $\begin{array}{l}\text { Diastolic Blood pressure } \\
4 \mathrm{~h}\end{array}$ & $\begin{array}{l}\text { Mean } \pm \text { SD } \\
\text { Range }\end{array}$ & $\begin{array}{c}60.50 \pm 10.20 \\
45-90\end{array}$ & $\begin{array}{c}65.00 \pm 10.67 \\
50-85\end{array}$ & -1.670 & 0.100 & NS \\
\hline $\begin{array}{l}\text { Diastolic Blood pressure } \\
6 \mathrm{~h}\end{array}$ & $\begin{array}{l}\text { Mean } \pm \text { SD } \\
\text { Range }\end{array}$ & $\begin{array}{c}63.17 \pm 9.96 \\
45-90\end{array}$ & $\begin{array}{c}66.67 \pm 9.03 \\
55-90\end{array}$ & -1.426 & 0.159 & NS \\
\hline
\end{tabular}

P-value >0.05: Non significant (NS); P-value <0.05: Significant (S); P-value $<0.01$ : highly significant (HS)

$\bullet:$ Independent t-test

Table (14): Comparison between HR regarding of the two blocks in the study.

\begin{tabular}{|c|c|c|c|c|c|c|}
\hline & $\begin{array}{l}\text { Dexmedetomidine } \\
\text { group }\end{array}$ & $\begin{array}{c}\text { Dexamethasone } \\
\text { group }\end{array}$ & \multirow[t]{2}{*}{ Test value• } & \multirow[t]{2}{*}{ P-value } & \multirow[t]{2}{*}{ Sig. } \\
\hline & & Mean \pm SD & Mean \pm SD & & & \\
\hline Heart Rate 0 & $\begin{array}{l}\text { Mean } \pm \text { SD } \\
\text { Range }\end{array}$ & $\begin{array}{c}77.53 \pm 7.04 \\
65-90\end{array}$ & $\begin{array}{c}79.03 \pm 5.83 \\
70-90\end{array}$ & -0.899 & 0.372 & NS \\
\hline Heart Rate 15 mins & \begin{tabular}{|l|}
$\begin{array}{l}\text { Mean } \pm \text { SD } \\
\text { Range }\end{array}$ \\
\end{tabular} & $\begin{array}{c}76.50 \pm 6.62 \\
66-88\end{array}$ & $\begin{array}{c}79.10 \pm 6.12 \\
68-90\end{array}$ & -1.579 & 0.120 & NS \\
\hline Heart Rate 45 mins & $\begin{array}{l}\text { Mean } \pm \text { SD } \\
\text { Range }\end{array}$ & $\begin{array}{c}75.13 \pm 6.97 \\
62-88\end{array}$ & $\begin{array}{c}78.23 \pm 6.72 \\
68-88\end{array}$ & -1.754 & 0.085 & NS \\
\hline Heart Rate 60mins & $\begin{array}{l}\text { Mean } \pm \text { SD } \\
\text { Range }\end{array}$ & $\begin{array}{c}73.97 \pm 6.77 \\
62-86\end{array}$ & $\begin{array}{c}76.83 \pm 6.44 \\
66-88\end{array}$ & -1.680 & 0.098 & NS \\
\hline Heart Rate $120 \mathrm{mins}$ & \begin{tabular}{|l|}
$\begin{array}{l}\text { Mean } \pm \text { SD } \\
\text { Range }\end{array}$ \\
\end{tabular} & $\begin{array}{c}73.20 \pm 6.95 \\
60-86\end{array}$ & $\begin{array}{c}75.73 \pm 5.78 \\
65-85\end{array}$ & -1.536 & 0.130 & NS \\
\hline Heart Rate $4 \mathrm{~h}$ & \begin{tabular}{|l|}
$\begin{array}{l}\text { Mean } \pm \text { SD } \\
\text { Range }\end{array}$ \\
\end{tabular} & $\begin{array}{c}73.67 \pm 6.83 \\
62-88 \\
\end{array}$ & $\begin{array}{c}77.13 \pm 5.26 \\
66-86 \\
\end{array}$ & -2.201 & 0.032 & $\mathrm{~S}$ \\
\hline Heart Rate $6 \mathrm{~h}$ & Mean \pm SD & $\begin{array}{l}74.03 \pm 6.92 \\
62-88\end{array}$ & $\begin{array}{l}77.63 \pm 5.90 \\
65-86\end{array}$ & -2.168 & 0.034 & $S$ \\
\hline
\end{tabular}

P-value >0.05: Non significant (NS); P-value <0.05: Significant (S); P-value $<0.01$ : highly significant (HS)

$\bullet:$ Independent t-test 
Table (15): Comparison between respiratory rate regarding of the two blocks in the study.

\begin{tabular}{|c|c|c|c|c|c|c|}
\hline & $\begin{array}{c}\text { Dexmedetomidine } \\
\text { group }\end{array}$ & $\begin{array}{c}\text { Dexamethasone } \\
\text { group }\end{array}$ & \multirow[t]{2}{*}{ Test value• } & \multirow[t]{2}{*}{ P-value } & \multirow[t]{2}{*}{ Sig } \\
\hline & & Mean \pm SD & Mean \pm SD & & & \\
\hline Respiratory Rate 0 & $\begin{array}{l}\text { Mean } \pm \text { SD } \\
\text { Range }\end{array}$ & $\begin{array}{c}12.40 \pm 1.13 \\
10-15 \\
\end{array}$ & $\begin{array}{c}12.37 \pm 0.89 \\
11-15 \\
\end{array}$ & 0.127 & 0.900 & NS \\
\hline Respiratory Rate $15 \mathrm{mins}$ & $\begin{array}{l}\text { Mean } \pm \text { SD } \\
\text { Range }\end{array}$ & $\begin{array}{c}12.40 \pm 1.13 \\
10-15\end{array}$ & $\begin{array}{c}12.30 \pm 0.75 \\
11-14\end{array}$ & 0.403 & 0.688 & NS \\
\hline Respiratory Rate $45 \mathrm{mins}$ & $\begin{array}{l}\text { Mean } \pm \text { SD } \\
\text { Range }\end{array}$ & $\begin{array}{c}12.40 \pm 1.13 \\
10-15\end{array}$ & $\begin{array}{c}12.23 \pm 0.68 \\
11-14\end{array}$ & 0.691 & 0.492 & NS \\
\hline Respiratory Rate $60 \mathrm{mins}$ & $\begin{array}{l}\text { Mean } \pm \text { SD } \\
\text { Range }\end{array}$ & $\begin{array}{c}12.50 \pm 1.15 \\
10-15\end{array}$ & $\begin{array}{c}12.13 \pm 0.75 \\
11-14\end{array}$ & -1.476 & 0.145 & NS \\
\hline Respiratory Rate $120 \mathrm{mins}$ & $\begin{array}{l}\text { Mean } \pm \text { SD } \\
\text { Range }\end{array}$ & $\begin{array}{c}12.45 \pm 1.09 \\
10-15\end{array}$ & $\begin{array}{c}12.33 \pm 0.80 \\
11-14\end{array}$ & -0.486 & 0.629 & NS \\
\hline Respiratory Rate $4 \mathrm{~h}$ & $\begin{array}{l}\text { Mean } \pm \text { SD } \\
\text { Range }\end{array}$ & $\begin{array}{c}12.42 \pm 1.33 \\
10-15\end{array}$ & $\begin{array}{c}12.25 \pm 0.89 \\
11-14\end{array}$ & -0.582 & 0.563 & NS \\
\hline Respiratory Rate $6 \mathrm{~h}$ & Mean \pm SD & $\begin{array}{l}12.48 \pm 1.52 \\
10-15\end{array}$ & $\begin{array}{l}12.29 \pm 1.60 \\
11-14\end{array}$ & -0.472 & 0.639 & NS \\
\hline
\end{tabular}

P-value >0.05: Non significant (NS); P-value <0.05: Significant (S); P-value< 0.01: highly significant (HS)

$\bullet:$ Independent t-test

Table (16): Comparison between SPO2 regarding of the two blocks in the study.

\begin{tabular}{|c|c|c|c|c|c|c|}
\hline & $\begin{array}{c}\text { Dexmedetomidine } \\
\text { group }\end{array}$ & $\begin{array}{c}\text { Dexamethasone } \\
\text { group }\end{array}$ & \multirow[t]{2}{*}{ Test value• } & \multirow[t]{2}{*}{ P-value } & \multirow[t]{2}{*}{ Sig. } \\
\hline & & Mean \pm SD & Mean \pm SD & & & \\
\hline SPO2 0 & $\begin{array}{l}\text { Mean } \pm \text { SD } \\
\text { Range }\end{array}$ & $\begin{array}{l}98.40 \pm 0.63 \\
98-99\end{array}$ & $\begin{array}{c}98.47 \pm 0.70 \\
97-99\end{array}$ & -0.455 & 0.651 & NS \\
\hline SPO2 $15 \mathrm{mins}$ & $\begin{array}{l}\text { Mean } \pm \text { SD } \\
\text { Range }\end{array}$ & $\begin{array}{l}98.33 \pm 0.53 \\
98-99\end{array}$ & $\begin{array}{c}98.57 \pm 0.63 \\
97-99\end{array}$ & 1.597 & 0.115 & NS \\
\hline SPO2 45mins & $\begin{array}{l}\text { Mean } \pm \text { SD } \\
\text { Range }\end{array}$ & $\begin{array}{l}98.65 \pm 0.79 \\
98-99\end{array}$ & $\begin{array}{c}98.59 \pm 0.80 \\
97-99\end{array}$ & -0.292 & 0.771 & NS \\
\hline SPO2 60mins & $\begin{array}{l}\text { Mean } \pm \text { SD } \\
\text { Range }\end{array}$ & $\begin{array}{l}98.49 \pm 0.69 \\
98-99\end{array}$ & $\begin{array}{c}98.70 \pm 0.77 \\
97-99\end{array}$ & 1.112 & 0.271 & NS \\
\hline SPO2 120mins & $\begin{array}{l}\text { Mean } \pm \text { SD } \\
\text { Range }\end{array}$ & $\begin{array}{c}98.90 \pm 0.80 \\
98-99\end{array}$ & $\begin{array}{c}98.85 \pm 0.69 \\
97-99\end{array}$ & -0.259 & 0.796 & NS \\
\hline SPO2 4h & Mean \pm SD & $\begin{array}{l}98.65 \pm 0.70 \\
98-99\end{array}$ & $\begin{array}{l}98.53 \pm 0.63 \\
97-99\end{array}$ & -1.279 & 0.206 & NS \\
\hline
\end{tabular}

P-value >0.05: Non significant (NS); P-value <0.05: Significant (S); P-value< 0.01: highly significant (HS)

$\bullet:$ Independent t-test

\section{DISCUSSION}

An effective, reliable block has shown to be provided by the application of US guided supraclavicular brachial plexus during upper extremity surgery, leading to a fewer complication, high satisfaction from patient and surgeon, reducing post operative analgesia and long time till the $1^{\text {st }}$ analgesia request as well as less hospital stay and less financial burden ${ }^{(12)}$.

In this study we aimed to compare the efficacy of dexmedetomidine versus dexamethasone as adjuvants on block characteristics (onset and duration of sensory and motor blockade, duration of postoperative analgesia, post operative pain score and sedation score).

The current study showed that the addition of dexmedetomidine to bupivacaine for supraclavicular brachial plexus block leads to prolongation of motor and sensory block duration, prolongation of duration of analgesia, more sedation in comparative to dexamethasone. onset of motor and sensory block was shorter in dexamethasone group than dexmedetomidine group.

Many studies had used dexmedetomidine as an adjuvant to LA in different regional and peripheral nerve blocks and found that it is an excellent choice in potentiating the local anesthetic effect ${ }^{(13)}$.

Choi et al and Brummett $\boldsymbol{e t}$ al. in their studies on administration of dexmedetomidine as an adjuvant to local anesthetics reported that the mechanism of the analgesic effect of dexmedetomidine is still not clear and may be multifactorial ${ }^{(14,15)}$. Possible mechanisms explained by Masuki et al., Yoshitomi $e t$ al. and Talke et $\boldsymbol{a l}$. were that dexmedetomidine induces 
vasoconstriction through an action on $\alpha 2$ adrenoceptors or it produces analgesia peripherally by reducing norepinephrine release and increasing the potassium conduction in $\mathrm{C}$ and A-delta neurons responsible for passage of pain stimulus, whereas it produces analgesia and sedation centrally by inhibition of substance $\mathrm{P}$ release in the nociceptive pathway at the level of the dorsal root ganglia and locus ceruleus $(16,17,18)$.

Marhofer et al. and Brummett et al. mentioned in their studies that the duration of analgesia achieved by dexmedetomidine may be in relation to block of the hyperpolarization-activated cation current (Ih current) which prevents the nerve from returning from a hyperpolarized state to resting membrane potential for subsequent firing and generation of a new action potential. This current seems to be more apparent in the unmyelinated $\mathrm{C}$ fibers (pain) than in A $\alpha$ fibres (motor). Therefore, blocking the $\left(\mathrm{I}_{\mathrm{h}}\right)$ current may have a more pronounced effect on pain than on motor response and this may explain the action of dexmedetomidine in prolongation of local anesthetics in peripheral nerve block ${ }^{(15,19)}$.

Mazy et al suggest that dexmedetomidine alone doesn't provide a significant motor and sensory block, but it potentiates local anesthetic action due to hyperpolarization activated cation $\left(\mathrm{I}_{\mathrm{h}}\right)$ current, not through $\alpha 2$ action, and similar to clonidine peripheral analgesia may be through release of an encephalin like substance.

In our study we found the addition of $(1 \mu \mathrm{g} / \mathrm{kg})$ of dexmedetomidine to $20 \mathrm{ml}$ bupivacaine $0.5 \%$ significantly prolonged the block duration as well as analgesia duration. These results is met with Marhofer et al, Swain et al, they found in their studies the addition of dexmedetomidine significantly prolonged block duration as well as duration of analgesia $^{(19,20)}$.

Swain et al. has studied the effect of addition of dexmedetomidine in supraclavicular, interscalene, cervical plexus and ulnar nerve blocks where dexmedetomidine has shown to increase quality and duration of analgesia of commonly used local anesthetics like ropivacaine and bupivacaine. An interesting study found that dexmedetomidine fared significantly better than clonidine when used as a adjuvant in supraclavicular blocks, the meta analysis examined primarily brachial plexus dose of 0.75 $\mu \mathrm{g} / \mathrm{kg}, 1.0 \mu \mathrm{g} / \mathrm{kg}$ and $100 \mu \mathrm{g}$ of dexmedetomidine ${ }^{(20)}$. Abdallah et al. review in their meta analysis the addition of dexmedetomidine in brachial plexus block at the axillary, supraclavicular, infraclavicular levels may prolong the duration of sensory block by 284 min, a relative increase of $76 \%$ compared with LA alone, and duration of motor block by $268 \mathrm{~min}$, with a relative increase of $87 \%$ compared with LA alone, and increase time of first analgesia request by 345 min, with a relative increase $70 \%$ compared with LA alone $^{(21)}$.

On the other hand, there were studies that had used dexamethasone as an adjuvant to LA regional or peripheral nerve blocks and resulted in longer sensory and motor blockade duration. This results met with Swain et al.and Liu et al. ${ }^{(20,22)}$.

Liu et al. Demonstrated that perineural dexamethasone (1,2 and $4 \mathrm{mg}$ ) significantly prolongs analgesia and motor block duration with $0.25 \%$ bupivacaine in supraclavicular brachial plexus nerve block. In addition, this study did not show statistically significant differences among different dosages of dexamethasone on analgesia duration and motor block prolongation ${ }^{(22)}$. Vieira et al. studied effects of $20 \mathrm{~mL}$ $0.5 \%$ bupivacaine on interscalene brachial plexus block in combination with 8-mg dexamethasone and without dexamethasone in control group and observed analgesia duration for 24.3 and 13.9 hours respectively ${ }^{(23)}$.

Biradar et al. in their study said in dexamethasone as adjuvant to bupivacaine in ultrasound guided supra clavicular block produced a relatively rapid onset of motor and sensory block ${ }^{(24)}$. Kishore et al. was observed the onset time of motor and sensory block was faster in dexamethasone group and clonidine group than control group in their study ${ }^{(25)}$.

But on other side Vieira et al. ${ }^{(23)}$ performed ultrasoundguided interscalene brachial plexus block in 88 patients scheduled for shoulder arthroscopy using $20 \mathrm{ml}$ of the local anesthetic mixture with dexamethasone adjuvant. There was no significant reduction in onset of sensory and motor blockade in dexamethasone group compared to control group. This discrepancy could be due to the difference in the local anesthetic volume and technique of block.

Possible explanations mentioned by Movafegh $\boldsymbol{e t}$ al. and Bastos $\boldsymbol{e t}$ al. were that it may be related to a degree of vasoconstriction occurred perineurally which results in a slow absorption of local anesthetics, suppression of the synthesis and secretion of inflammatory mediators, reducing the transmission in unmyelinated C-fiber, through immune-suppressive effects or local effect on the nerve ${ }^{(26,27)}$. Abdallah et al. believe that analgesic properties of dexamethasone are the result of their systemic effect ${ }^{(28)}$. Movafegh et al. and Bastos et al. suggest local action on nerve fiber and systemic action both potentiate dexamethasone analgesic properties $^{(26,27)}$.

Regarding demographic data and operative characteristics there were no statistically significant difference between the two groups.

Regarding hemodynamic parameters (HR,SBP,DBP,RR,SPO ${ }_{2}$ ) the results were not statistically significant which is met with the results 
of Abdelnaim et al. Mangal et al. and Bharti et al. ${ }^{(29,30,31)}$. Meanwhile heart rate decrease in dexmedetomidine group at $15,30,45,60,90,120 \mathrm{~min}$ within normal value. Esmaoglu et al. reported that the addition of $100 \mu \mathrm{g}$ dexmedetomidine to $0.5 \%$ levobupivacaine caused bradycardia (HR $<50$ beats/min) in 7 of 30 patients. In the present study, although we noticed lower HRs (50-60 beats/min) in the dexmedetomidine group, none of our patients had bradycardia or hypotension. This may be because we used a smaller dose of dexmedetomidine ${ }^{(32)}$. On the other side Almarakbi et al. recorded significant fall in the heart rate 60 minutes following administration of $(1 \mu \mathrm{g} / \mathrm{kg})$ dexmedetomidine in TAP block opposite to the control group and this effect persisted 4 hours without any hemodynamic instability. This is a known side effect of the drug and might be related to the post-synaptic activation of central $\alpha 2$ adrenoceptors ${ }^{(33)}$.

Abdallah et al. reviewed in their meta analysis the incidence of hypotension was similar in dexmedetomidine group and control group, but incidence of brady cardia was higher in patients receiving dexmedetomidine ${ }^{(28)}$.

Regarding sedation score In our study, sedation scores were higher in patients receiving dexmedetomidine compared to the dexamethasone group. Intraoperatively, sedation was observed in 17patient from 30 patient from 15 minutes to 120 minutes' time point in the dexmedetomidine group but the patient was easily arousable. The modified Ramsay sedation score for dexmedetomidine group was either $3 / 6$ or $4 / 6$ in a majority of the cases, while that for dexamethasone group it was $2 / 6$.

Abdallah et $\boldsymbol{a l}$. was evaluated sedation in 4 trials that examined intrathaecal injection of dexmedetomidine, they reported higher sedation level in a group of patient receiving high dose of dexmedetomidine $(15 \mathrm{~g})$ while the other reported no difference.

No patient experienced airway compromise or required airway assistance because of sedation. This results is met with Mangal et al, Abdelnaim et al. ${ }^{(29,30)}$.

On other hand Marhofer et al, said the addition of dexmedetomidine to ropivacaine does not produce sedation we suggest this is because low dose of dexmedetomidine $(20 \mu \mathrm{g})$ used by Marhofer and colleauges.

Regarding post operative pain score (numeric rating scale of pain) in our study. There were no statistical significant difference between the two groups. There were no recorded complications related to the block techniques or to the drugs in all patients in the form of nausea and vomiting hemodynamic instability, injury to underlying structures, hematoma formation, infection or local anesthetic toxicity. Recep et al. ${ }^{(34)}$ mentioned in their study nausea and vomiting was seen in one case, dry mouth was seen in one case in their dexmedetomidine group.

\section{CONCLUSION}

Regarding

comparison

between

dexmedetomidine and dexamethasone as an additive to bupivacaine in ultrasound guided supraclavicular block, it could be concluded that the addition of dexmedetomidine to bupivacaine prolong the time of block and analgesia duration longer than dexamethasone, but the onset of block was shorter when dexamethasone was added to bupivacaine.

\section{REFERENCES}

1. Jarbo K, Batra YK, Panda NB (2005): Brachial plexus block with midazolam and bupivacaine improves analgesia. Can J Aesth., 52:822-826

2. Basin JE, Masson C, Bruelle $P$ et al. (1997): The addition of opioids to local anesthetics in brachial plexus block: the comparative effects of morphine, buprenorphine and sufentanil. Anesthesia, 52:858-862.

3. Kesimci E, Izdes S, Gozdemir M et al. (2007): Tramadol does not prolong the effect of ropivacaine 7.5 $\mathrm{mg} / \mathrm{ml}$ for axillary brachial plexus block. Acta Anaesthesiol Scand., 51:736-741.

4. Amlan S, Deb SN, Seelora S, Devi PS (2017): Adjuvants to local anesthetics: Current understanding and future trends. World J Clin Cases, 5(8): 307-323

5. Brummett CM, Norat MA, Palmisano JM et al. (2008): Perineural administration of dexmedetomidine in combination with bupivacaine enhances sensory and motor blockade in sciatic nerve block without inducing neurotoxicity in rat. Anesthesiology, 109:502-511.

6. Kaygusuz K, Kol IO, Duger C et al. (2012): Effects of adding dexmedetomidine to levobupivacaine in axillary brachial plexus block. Curr Ther Res Clin Exp., 73:103111.

7. Gandhi R, Shah A, Patel I (2012): Use of dexmedetomidine along with bupivacaine for brachial plexus block. National J Med Res., 2:67-69.

8. Kaygusuz K, Kol IO, Duger C et al. (2012): Effects of adding dexmedetomidine to levobupivacaine in axillary brachial plexus block. Curr Ther Res Clin Exp., 73:103111.

9. Benzon HT, Chew TL, McCarthy RJ et al. (2007): Comparison of the particle sizes of different steroids and the effect of dilution: A review of the relative neurotoxicities of the steroids. Anesthesiology, 106:331338.

10. Vieira PA, Pulai I, Tsao GC et al. (2010): Dexamethasone with bupivacaine increases duration of analgesia in ultrasound-guided interscalene brachial plexus blockade. Eur J Anaesthesiol., 27: 285-288.

11.Biradar PA, Kaimar P, Gopalakrishna K (2013): Effect of dexamethasone added to lidocaine in supraclavicular brachial plexus block: A prospective, randomised, double-blind study. Indian $\mathrm{J}$ Anesth., 57:180-184. 
12. More P, Basavaraja, Laheri V (2015): A comparison of dexmedetomidine and clonidine as an adjuvant to local anaesthesia in supraclavicular brachial plexus block for upper limb surgeries. JMR., 1:142-147.

13. Kanazi GE, Aouad MT, JAbbour-Khoury SL et al. (2006): Effects of low dose Dexmedetomidine or clonidine on characteristics of spinal block. Acta Anaesthesiol Scand., 50:222-227.

14. Choi S, Rodseth R, McCartney CJ (2014): Effects of dexamethasone as a local anaesthetic adjuvant for brachial plexus block: a systematic review and metaanalysis of randomized trials. Br J Anaesth., 112(3):42739.

15. Brummett CM, Norat MA, Palmisano JM, Lydic R (2008): Perineural administration of dexmedetomidine in combination with bupivacaine enhances sensory and motor blockade in sciatic nerve block without inducing neurotoxicity in rat. Anesthesiology, 109:502-511

16. Masuki S, Dinenno FA, Joyner MJ et al. (2005): Selective alpha2- adrenergic properties of dexmedetomidine over clonidine in the human forearm. $\mathbf{J}$ Appl Physiol., 99:587-592.

17. Yoshitomi T, Kohjitani A, Maeda S et al. (2008): Dexmedetomidine enhances the local anesthetic action of lidocaine via an alpha-2A adrenoceptor. Anesth Analg., 107:96-101.

18. Talke P, Lobo E, Brown R (2003): Systemically administered alpha2- agonist-induced peripheral vasoconstriction in humans. Anesthesiology, 2003; 99: 65-70.

19. Marhofer D, Kettner SC, Marhofer S et al. (2012): Dexmedetomidine as an adjuvant to ropivacaine prolongs peripheral nerve block: A volunteer study. Br J Anesth., 15:438-442.

20.Swain A, Nag DS, Sahu S, Samaddar DP (2017): Adjuvant to local anesthesics:Current understanding and future trends. World J Clin Cases., 5(8):307-311.

21. Abdallah FW, Brull R (2013): Facilitatory effects of perineural dexmedetomidine on neuraxial and peripheral nerve block: a systematic review and meta-analysis. British Journal of Anaesthesia, 110 (6): 915-925.

22.Liu J, Richman KA, Grodofsky SR, Bhatt S, Huffman GR, Kelly JD, Glaser DL, Elkassabany N (2015): Is there a dose response of dexamethasone as adjuvant for supraclavicular brachial plexus nerve block? A prospective randomized double-blinded clinical study. J Clin Anesth., 27:237-242.

23. Vieira PA, Pulai I, Tsao GC et al. (2010): Dexamethasone with bupivacaine increases duration of analgesia in ultrasound-guided interscalene brachial plexus blockade. Eur J Anaesthesiol., 27: 285-288.

24. Biradar PA, Kaimar P, Gopalakrishna K (2013): Effect of dexamethasone added to lidocaine in supraclavicular brachial plexus block: A prospective, randomised, double- blind study. Indian J Anaesth., 57:180-184.
25.Hari K, Rajagopal P, Binu PS et al. (2015): Comparison between $0.5 \%$ Bupivacaine-Dexamethasone Combination \& $0.5 \%$ Bupivacaine - Clonidine Combination in Brachial Plexus Blocks by Supraclavicular Approach. Journal of Evidence based Medicine and Healthcare, 2(15):3016-3024

26. Movafegh A, Razazian M, Hajimaohamadi F et al. (2006): Dexamethasone added to lidocaine prolongs axillary brachial plexus blockade. Anesth Analg., 102(1):263-267.

27. Bastos LF, Medeiros DC, Vieira RP et al. (2012): Intraneural dexamethasone applied simultaneously to rat sciatic nerve constriction delays the development of hyperalgesia and allodynia. Neurosci Lett., 510: 20-23.

28. Abdallah FW, Brull R (2013): Facilitatory effects of perineural dexmedetomidine on neuraxial and peripheral nerve block: a systematic review and meta-analysis. British Journal of Anaesthesia, 110 (6): 915-925.

29. Abdelnaim HE, Mohamed NN et al. (2018): Comparison between bupivacaine- dexmedetomidine mixture and bupivacaine-magnesium mixture when used for wound infiltration before skin incision in surgeries for hernia repair regarding their intraoperative and postoperative analgesic effects. Ain-Shams Journal of Anesthesiology, 10:10

30. Mangal V, Mistry T, Sharma G, Kazim M, Ahuja N, Kulshrestha A (2018): Effects of dexmedetomidine as an adjuvant to ropivacaine in ultrasound-guided supraclavicular brachial plexus block: A prospective, randomized, double-blind study. J Anaesthesiol Clin Pharmacol., 34:357-61.

31. Bharti N, Sardana DK, Bala I (2015): The Analgesic Efficacy of Dexmedetomidine as an Adjunct to Local Anesthetics in Supraclavicular Brachial Plexus Block: A Randomized Controlled Trial. Anesth Analg., 121:1655-1660.

32. Esmaoglu A, Yegenoglu F, Akin A et al. (2010): Dexmedetomidine added to levobupivacaine prolongs axillary brachial plexus block. Anesth Analg., 111:15481551.

33. Almarakbi WA, Kaki AM (2014): Addition of dexmedetomidine to bupivacaine in transversus abdominis plane block potentiates post- operative pain relief among abdominal hysterectomy patients: A prospective randomized controlled trial. Saudi J Anesth., 8:161-166.

34. Recep A, Akin A, Biçer C et al. (2009): Comparison of the effects of dexmedetomidine versus fentanyl on airway reflexes and hemodynamic responses to tracheal extubation during rhinoplasty: A double-blind, randomized, controlled study. Current therapeutic research,

70(3):209-20. 